\title{
Synthesis and mechanical properties of polybenzimidazole nanocomposites reinforced by vapor grown carbon nanofibers
}

\author{
Li Zhang ${ }^{1}$, Qing-Qing $\mathrm{Ni}^{1}$, Akihiko Shiga ${ }^{2}$, Yaqin $\mathrm{Fu}^{3}$, Toshiaki Natsuki ${ }^{1}$ \\ ${ }^{1}$ Faculty of Textile Science \& Technology, Shinshu University, Japan \\ ${ }^{2}$ AZ Electronic Materials (Japan) K.K., PBI Business Production \& Technology Group \\ ${ }^{3}$ Key Laboratory of Advanced Textile Materials and Manufacturing Technology \\ Ministry of Education, Zhejiang Sci-Tech University, Hangzhou 310018， P.R. China
}

\begin{abstract}
Polybenzimidazole (PBI) nanocomposites containing 0.5-5 wt\% vapor grown carbon nanofibers (VGNFs) were successfully synthesized by solvent evaporation method. Fracture morphology examination confirmed the uniform dispersion of VGNFs in the matrix. The mechanical properties of neat PBI and the nanocomposites were systematically measured by tensile test, dynamic mechanical analysis (DMA), hardness measurement and friction test. Tensile tests revealed that Young's modulus increased by about $36.7 \%$ at $2 \mathrm{wt} \%$ VGNFs loading, and further
\end{abstract}

Correspondence to: Qing-Qing Ni; e-mail: niqq@ shinshu-u.ac.jp modulus growth was observed at higher filler loadings. DMA studies showed that the nanocomposites have higher storage modulus than neat $\mathrm{PBI}$ in the temperature range of 30-350 ${ }^{\circ} \mathrm{C}$, holding storage modulus larger than 1.54 GPa below $300{ }^{\circ} \mathrm{C}$. Outstanding improvement of hardness was achieved for polybenzimidazole upon incorporating 2 wt $\%$ of VGNFs. The results of friction test showed that coefficient of friction of polybenzimidazole nanocomposites decreased with VGNFs content compared to neat PBI.

\section{INTRODUCTION}

Polybenzimidazole (PBI) commercialized by the Celanese Corporation in 1983 is an aromatic heterocyclic polymer that is resistant to strong acids, bases, and high temperatures [1]. PBI are widely used in firefighter protective clothing, astronaut flight suits due to its excellent thermal stability [1,2], and used as insulating layer of semiconductor industry due to its high volume resistivity. Recently, phosphoric acid doped PBI membrane has emerged as a promising candidate for polymer electrolyte in high temperature proton exchange membrane fuel cell (PEMFC) [3, 4]. Because of excellent film forming capability, PBI has been used as coating material since the 1990s $[5,6]$. Development of technologies leads unavoidably to increasingly large demands on PBI materials with higher mechanical performance. It is well known that the mechanical performance of polymer materials can be effectively improved by introducing a suitable amount of inorganic reinforcement such as carbon nanofibers to form composite materials $[7,8]$. Therefore, research on the preparation and mechanical properties of PBI nanocomposites is of great importance.

Polymer nanocomposites that are reinforced by inorganic nanoparticles exhibit astonishing improvement in mechanical properties compared to the matrix. Normally, the fillers of the nanocomposites are layered silicate [9], clay [10, 11] and metal oxide nanoparticles [12, 13]. Recently, Carbon nanotubes (CNTs) and Carbon nanofibers 
(CNFs) are considered as the ideal reinforcement fillers for preparing nanocomposites owing to their outstanding mechanical characteristics, extremely large interfacial contact area, high aspect ratio, and low mass density [14-18]. Some literatures have been reported on the preparation and properties of polymer nanocomposites reinforced by CNTs or CNFs, including Polyimide/MWCNTs [14], PTFE/CNFs [15], Epoxy/VGCFs [16], PP/CNFs [17], and Polyol/CNFs [18]. However, polybenzimidazole nanocomposites have few reports up to now.

In this paper, the polybenzimidazole (PBI) nanocomposites reinforced by vapor grown carbon nanofibers were successfully synthesized by the solvent evaporation method. Compared to the neat PBI material, the nanocomposites exhibited much higher mechanical performance. It is reasonable to believe that the VGNFs-reinforced PBI nanocomposites have promising application in advanced technologies.

\section{EXPERIMENTAL}

Materials. The polymer matrix used in this study was PBI (AZ Electronic materials Japan K.K.) and was used in the form of PBI matrix resin solution (PBI dissolved in DMAc solution with the concentration of $10 \%$ ). The weight-average molecular weight, $M_{w}$, was $130000 \mathrm{Da}$, and the number-weight molecular weight, $\mathrm{M}_{\mathrm{n}}$, was 70000 Da. The VGNFs were provided by Showa Denko (Japan) with a diameter of $80 \mathrm{~nm}$ and length of 10-100 $\mathrm{\mu m}$ synthesized by the gas phase method. As-received VGNFs were purified and oxidized by boiling in concentrated $\mathrm{HNO}_{3}$ for $4 \mathrm{~h}$, according to literature methods [19]. This procedure was carried out in order to remove the rest of amorphous impurities. N, N, Dimethylacetamide (DMAc) was supplied by Wako pure chemical industries and used without any further purification.

Preparation of PBI/VGNFs nanocomposites. The acid treated VGNFs were dispersed in DMAc solution by a combined mixing (ultrasonic mixing (100 W, $45 \mathrm{KHz}$ ) and mechanical mixing) for 6 hours. Then the PBI matrix resin solution was added to the VGNFs suspension and combined mixing process continued for another 6 hours. The mixture was then cast in a mold and put into a furnace at $90{ }^{\circ} \mathrm{C}$ for 8 hours to evaporate the solvent and produce uniform sheet. Finally the sheet was compacted by a hot pressing machine at a pressure of $5 \mathrm{MPa}$, and held the temperature at $100{ }^{\circ} \mathrm{C}, 150$ ${ }^{\circ} \mathrm{C}, 200{ }^{\circ} \mathrm{C}, 250{ }^{\circ} \mathrm{C}, 300{ }^{\circ} \mathrm{C}$ and $350{ }^{\circ} \mathrm{C}$ for an hour respectively. The nanocomposites sheet was prepared with contents of $0.5,1,2$ and 5 wt $\%$ of VGNFs.

Characterizations. Raman spectroscopy measurements were carried out on a HoloLab series 5000 Raman spectroscope (Kaiser optical systems, INC.) furnished with a $514 \mathrm{~nm}$ laser excitation. Tensile properties were performed on a JIS K6251 universal testing machine (RTC 1250A A\&D Co. Ltd, Japan) at $0.5 \mathrm{~mm} / \mathrm{min}$ and $25^{\circ} \mathrm{C}$. Tensile specimens were cut from sheet samples according ISO 527-3. Five specimens of each material were tested for reproducibility. The morphology of the VGNFs and fracture surface after tensile test were observed using filed emission scanning electron microscopy (S-5000, Hitachi, Japan). The storage modulus of the resulting nanocomposites was acquired with the aid of Dynamic mechanical analyzer (DVA-225, ITK Co. Ltd., Japan). The dimension of the specimens was $20 \mathrm{~mm} \times 5 \mathrm{~mm} \times 0.1 \mathrm{~mm}$ (length $\times$ width $\times$ thickness). The scanning ranged from $30{ }^{\circ} \mathrm{C}$ to $350{ }^{\circ} \mathrm{C}$ at a heating rate of $10{ }^{\circ} \mathrm{C} / \mathrm{min}$ and a frequency of $1 \mathrm{~Hz}$. Hardness was measured using a Vickers hardness testing machine (Akashi microhardness tester, Model-MVK Type), with a pyramidal diamond indenter and a load of $0.2 \mathrm{~kg}$. The indentation diagonal lengths were 
measured by an optical microscope, and using the average of five indentions per specimen. Vickers hardness values were calculated according to the expression of $H v=1.854\left(P / d^{2}\right)$, where $P$ is load $(\mathrm{kg})$ and $d$ is the average diagonal length $(\mathrm{mm})$. Then the data was converted to SI units. Tribological property of PBI/VGNFs nanocomposites was evaluated by using the ball-on-disc rotating friction player FRP-2000 (Rhesca Ltd. Japan). The ball (stainless steel, a diameter of $9.7 \mathrm{~mm}$ ) was ultrasonically cleaned in ethanol for $5 \mathrm{~min}$ and dried in air prior to test. The disc with an area of $10 \mathrm{~mm}^{2}$ and a thickness of $0.1 \mathrm{~mm}$ was made of neat PBI and PBI/VGNFs nanocomposites. The rotating velocity was $71.6 \mathrm{rpm}$ with rotating radius of $4 \mathrm{~mm}$. Friction tests were carried out at dry ambient condition with a load of $5 \mathrm{~N}$ over a period of $20 \mathrm{~min}$.

\section{RESULTS AND DISCUSSION}

Typical SEM image of as-received VGNFs is shown in Fig. 1(a) and represents the highly entangled morphology with diameter around $80 \mathrm{~nm}$ and amorphous impurities. Comparison of SEM images of as-received VGNFs with acid purified VGNFs (Fig. 1(b)) shows obvious reduction of amorphous nanoparticles. Raman measurements (see Fig. 2) of the treated VGNFs also indicate apparent decrease of amorphous carbon according to the reduction of disorder mode band intensity ( $\mathrm{D}$ band).

Tensile stress-strain curves of neat PBI and PBI/VGNFs nanocomposites are presented in Fig. 3. The neat PBI stress-strain curve exhibits an obvious nonlinear region starting at about $120 \mathrm{MPa}$, which likely corresponds to the micro cracks and partial damage of molecular chains during the large elongation process. The ultimate strain of neat PBI is about $10.2 \%$. However, the nonlinear region in the stress-strain curves of nanocomposites decreases rapidly with the increment of VGNFs content. The Young's modulus and the tensile strength obtained from these tests are plotted in Fig. 4. VGNFs incorporation has considerable impact on PBI tensile properties; apparent Young's modulus increase by $36.7 \%$ from $2.59 \mathrm{GPa}$ to $3.13 \mathrm{GPa}$ at $0.5 \mathrm{wt} \%$ VGNFs loading and further modulus enhancement continues up to $3.59 \mathrm{GPa}$ at $5 \mathrm{wt} \%$. Tensile strength of the nanocomposites has a relative moderate enhancement until the VGNFs loading up to $2 \mathrm{wt} \%$ with $25.6 \%$ improvement from $124.7 \mathrm{MPa}$ to $156.7 \mathrm{MPa}$. However a sudden decrease happens at $5 \mathrm{wt} \%$ loading which maybe results from not only the increase of the probability of VGNFs agglomeration that is inevitable at such a high concentration but also the considerable decrease of plastic range in nanocomposites.

Scanning electron microscopy was conducted to analyze the morphology of the fracture surface after tensile test and the dispersion of VGNFs in the polymer matrix. Figure 5 shows the SEM results of these nanocomposites. The fracture surface of neat PBI is smooth with many "river like" patterns, which may be caused by the ductile deformation of PBI polymer. The fracture surfaces of nanocomposites show the three dimensional deformation patterns rather than a smooth plane like neat PBI. More deformation resistance can be obtained when the crack direction is changed due to the presence of VGNFs, which leads to increase the Young's modulus and tensile strength of PBI/VGNFs nanocomposites.

Nanoparticles are difficult to disperse uniformly in polymer matrices, especially nanotubes and/or nanofibers due to a strong tendency to agglomerate caused by their high surface areas. In the present study, no obvious agglomerations are found in the specimens of nanocomposites containing $0.5,1$ and $2 \mathrm{wt} \%$ due to intense mixing. 
However, a few agglomeratation phenomena are found in nanocomposites with $5 \mathrm{wt} \%$, which can be used to explain the sharp decrease in the tensile strength. Meanwhile, some VGNFs pulled out from polymer matrix are found on the fracture surfaces, which imply that the interface adhesion between VGNFs and the PBI matrix needs to be improved. This can be alleviated by adopting effective treatment of interface between the PBI and VGNFs, which will be discussed in the future.

In order to investigate the relationship between the storage modulus and temperature, the DMA was studied, and the corresponding storage modulus $\left(E^{\prime}\right)$ and temperature behavior are shown in Fig. 6. Addition of the VGNFs can increase the level of the storage modulus $E^{\prime}$ in the whole glassy region, with the highest value observed for the filler content of $5 \mathrm{wt} \%$. Table 1 compares the values of the storage modulus of PBI and PBI/VGNFs nanocomposites. The values of the nanocomposites appear much higher than that of the PBI matrix over the entire temperature range measured due to the efficient load transfer from polymer to VGNFs. The $E^{\prime}$ values of the nanocomposites with $2 \mathrm{wt} \%$ VGNFs loading improve by $84.0 \%$ (from 3.07 to $5.65 \mathrm{GPa}$ ) at $30{ }^{\circ} \mathrm{C}$, by $104.6 \%$ (from 1.30 to $2.66 \mathrm{GPa}$ ) at $300{ }^{\circ} \mathrm{C}$. On the other hand, the reduction of $E^{\prime}$ over the entire temperature range measured can be divided into two steps; below $300{ }^{\circ} \mathrm{C}$, there is a slow reduction in $E^{\prime}$ associated with gentle chain segmental motion of PBI molecules in the nanocomposites, and for the range between $300^{\circ} \mathrm{C}$ and $350{ }^{\circ} \mathrm{C}$, which belongs to a part of the glass transition region $\left(\mathrm{Tg}=429^{\circ} \mathrm{C}\right.$, data from $\mathrm{AZ}$ Electronic materials Japan K.K.), the sharp reduction in $E^{\prime}$ was observed because of acceleration of the chain segmental motion which is inevitable. In a word, all nanocomposites have exceptional thermal stability and hold the storage modulus larger than $1.54 \mathrm{GPa}$ below $300{ }^{\circ} \mathrm{C}$.

Hardness measurements were performed on well polished neat PBI and PBI/VGNFs nanocomposites samples, and the results are shown in Fig. 7. Vickers hardness of neat PBI is observed to be around $329.3 \mathrm{MPa}$ and it increases apparently after adding VGNFs. The hardness increases to $439.0 \mathrm{MPa}$ for the content of $2 \mathrm{wt} \%$ with about $33.3 \%$ improvement compared to neat PBI. This may be brought about by the uniform distribution of the VGNFs throughout the polymer matrix and the associated entanglement with the polymer chains.

The coefficient of friction is an important factor in understanding friction behavior. The friction curve of neat PBI (see Fig. 8) reveals that the neat PBI has a high value of coefficient of friction at approximately 0.24 , and it decreases sharply when the VGNFs content is lower than $1 \mathrm{wt} \%$. Then the coefficient of friction decreases slowly with increasing VGNFs content to $5 \mathrm{wt} \%$. The lower coefficient of friction of about 0.16 with $33.3 \%$ decrease is gotten at $2 \mathrm{wt} \%$ VGNFs loading. Thus, it is effective to reduce the coefficient of friction after adding VGNFs, and the incorporation of VGNFs in nanocomposites may act as a source of solid lubricant, as well as reinforcement materials.

\section{CONCLUSIONS}

In this study, polybenzimidazole nanocomposites reinforced by vapor grown carbon nanofibers were successfully synthesized by using a solvent evaporation method. Microscopic analysis showed that VGNFs were dispersed well in PBI matrix. Tensile test showed that the Young's modulus and the tensile strength were improved obviously compared with neat PBI with about $36.7 \%$ and $25.6 \%$ enhancement respectively at 2 
wt\% VGNFs loading. Dynamic mechanical analysis showed that the storage modulus increased by the incorporation of VGNFs and all the nanocomposites held the storage modulus larger than $1.54 \mathrm{GPa}$ below $300{ }^{\circ} \mathrm{C}$. After integrating VGNFs into PBI polymer matrix, the hardness also enhanced apparently. Compared with the neat PBI, the coefficient of friction in the nanocomposite of $2 \mathrm{wt} \%$ content of VGNFs decreased obviously by $30 \%$. As a consequence, VGNFs are very promising fillers to improve the mechanical properties of PBI polymer.

\section{ACKNOWLEDGMENTS}

This work was supported by Grant-in-Aid for Global COE Program by the Ministry of Education, Culture, Sports, Science, and Technology, Japan.

\section{REFERENCES}

1. J.R. Klaehn, T.A. Luther, C.J. Orme, M.G. Jones, A.K. Wertsching and E.S. Peterson, Macromoleculues, 40, 7487 (2007).

2. R.L. Barker, S.C. Guerth, R.V. Grimes and H. Hamouda, Text. Res. J., 76, 27 (2006).

3. Q.F. Li, C. Pan, J.O. Jensen, P. Noye and N.J. Bjerrum, Chem. Mater., 19, 350 (2007).

4. D. Mecerreyes, H. Grande, O. Miguel, E. Ochoteco, R. Marcilla and I.Cantero, Chem. Mater., 16, 604, (2004).

5. Y. Onishi, A. Maeno, H. Tanigawa, K. Iura, T. Ito, M. Nagata and T. Sasuga, Cryogenics, 35, 795 (1995).

6. S. Scianna, K.D. Berger and G. Schmitt, Kunststoffe, 82, 678 (1992).

7. G.G. Tibbetts, M.L. Lake, K.L. Strong and B.P. Rice, Compos. Sci. Technol., 67, 1769 (2008).

8. T. Hayashi, Y.A. Kim, T. Natsuki and M. Endo, ChemPhysChem, 8, 999 (2007).

9. H. Acharya, T. Kuila, S.K. Srivastava and A.K. Bhowmick, Polym. Compos., 29, 443 (2008).

10. J.M. Lee and D.S. Kim, Polym. Compos., 28, 325 (2007).

11. V. Causin, M.L. Carraro, C. Marega, R. Saini, S. Campestrini and A. Marigo, J. Appl. Polym. Sci., 109, 2354 (2008).

12. J. Wang and X.Y. Ni, J. Appl. Polym. Sci.,108, 3552 (2008).

13. Z.M. Dang, Y.Q. Lin, H.P. Xu, C.Y. Shi, S.T. Li and J.B. Bai, Adv. Funct. Mater., 18, 1 (2008).

14. T.C. Mo, H.W. Wang, S.Y. Chen and Y.C. Yeh, Polym. Compos., 29, 451 (2008).

15. Y.J. Shi, X. Feng, H.Y. Wang, X.H. Lu and J.Y. Shen, J. Appl. Polym. Sci., 104, 2430 (2007).

16. Y.K. Choi, K. Sugimoto, S.M. Song, Y. Gotoh, Y. Ohkoshi and M. Endo, Carbon, 43, 2199 (2005).

17. G. Sui, W.H. Zhong, M.A. Fuqua and C.A. Ulven, Macromol. Chem. Phys., 208, 1928 (2007).

18. S.M. Rhodes, B. Higginsa, Y.J. Xua and W.J. Brittaina, Polymer, 48, 1500 (2007).

19. P.V. Lakshminarayanan, H. Toghiani and C.U. Pittman Jr., Carbon, 42, 2433 (2004). 


\section{Figure captions}

Fig. 1 SEM images of the as-received VGNFs (a) and the acid treated VGNFs (b).

Fig. 2 Raman spectra of as-received VGNFs (a) and the acid treated VGNFs (b).

Fig. 3 Stress-strain curves of neat PBI and the nanocomposites.

Fig. 4 Comparison of Young's modulus and tensile strength for neat PBI and the nanocomposites.

Fig. 5 SEM images taken from the fractured surface of tensile specimens: (a) neat PBI; (b) $\mathrm{PBI}+0.5 \mathrm{wt} \%$; (c) PBI + $1 \mathrm{wt} \%$; (d) PBI + $2 \mathrm{wt} \%$.

Fig. 6 Comparison of the storage modulus for neat PBI and the nanocomposites.

Fig. 7 Variations of Vickers hardness with VGNFs content.

Fig. 8 Variations of coefficient of friction with VGNFs content. 


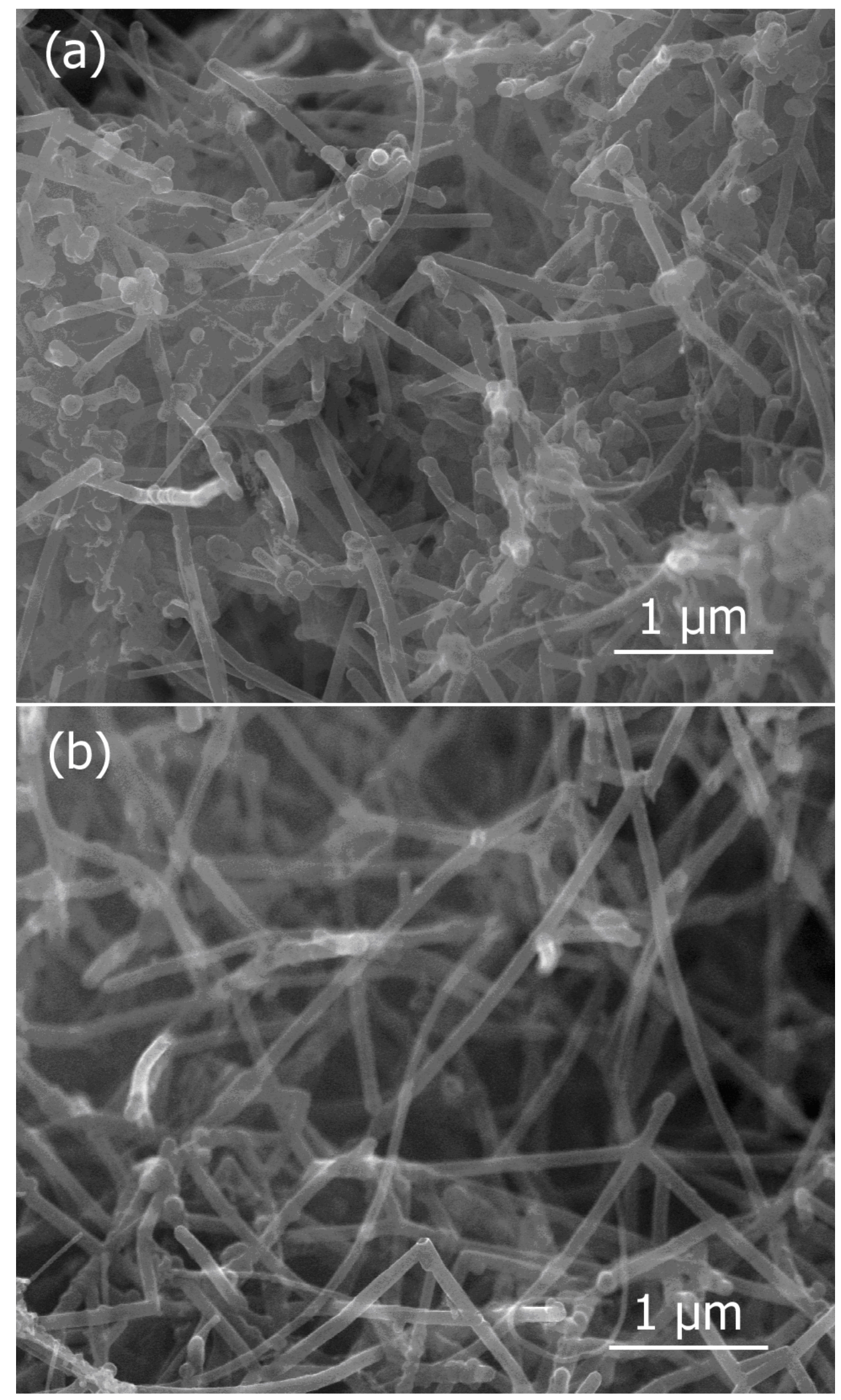

Fig. 1 


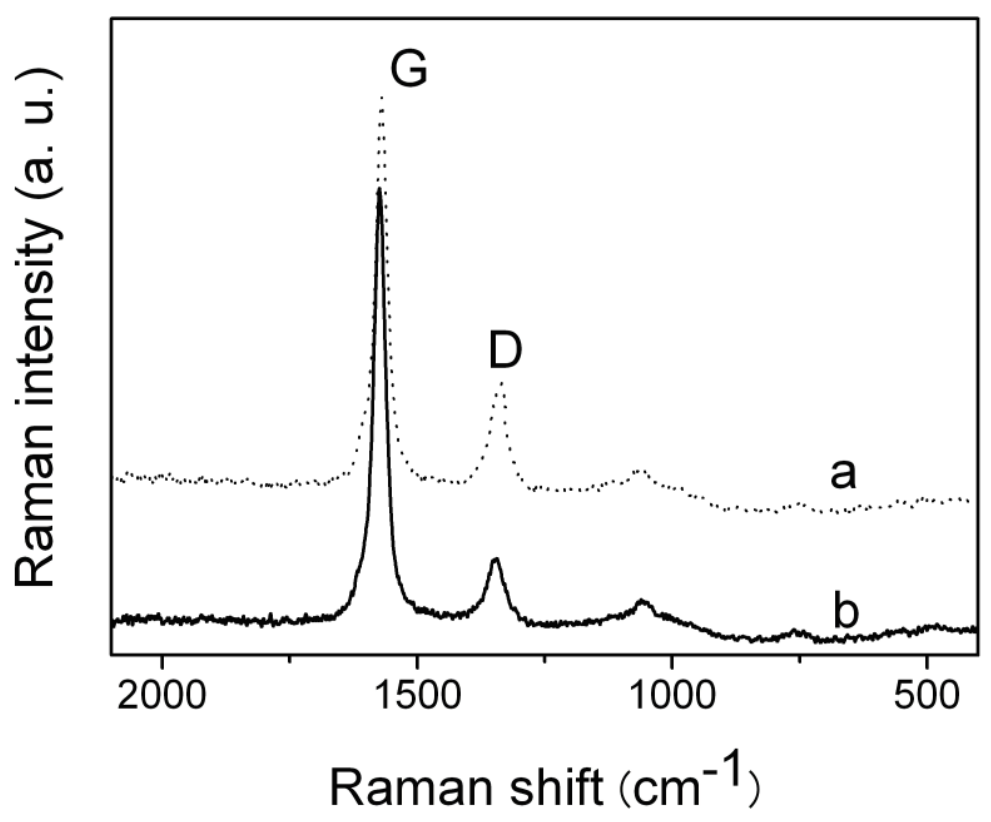

Fig. 2 


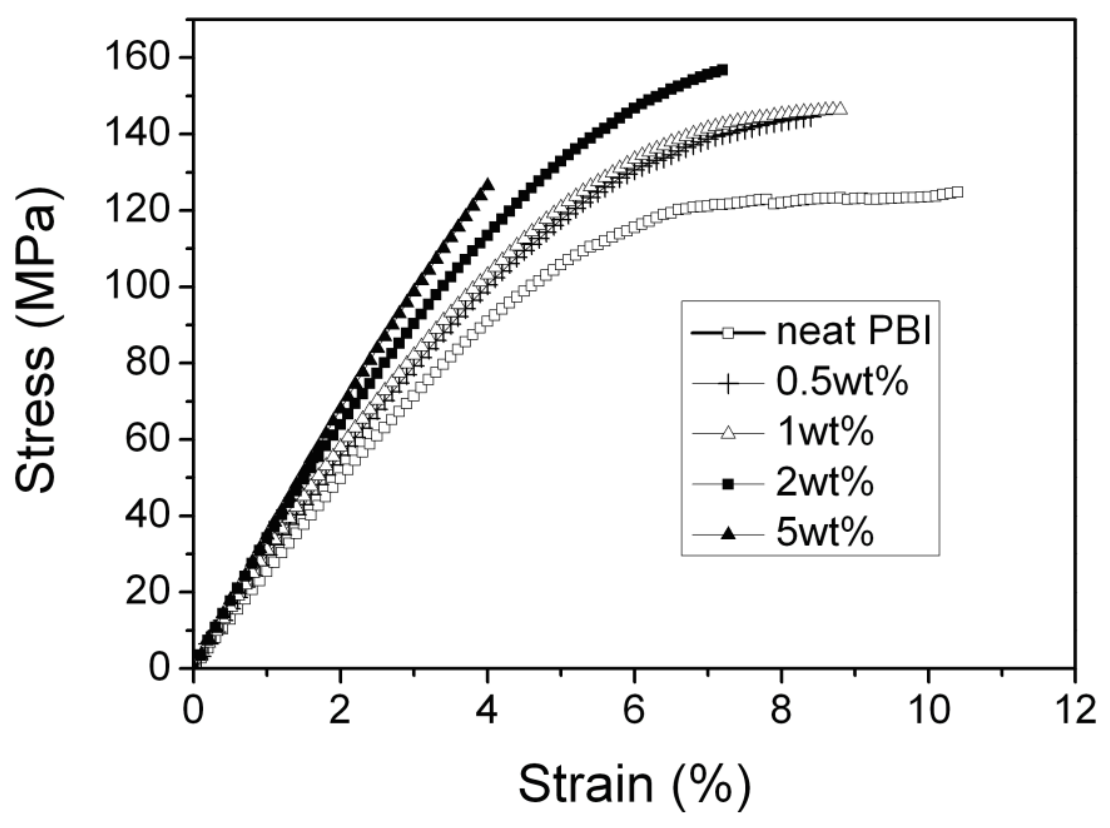

Fig. 3 


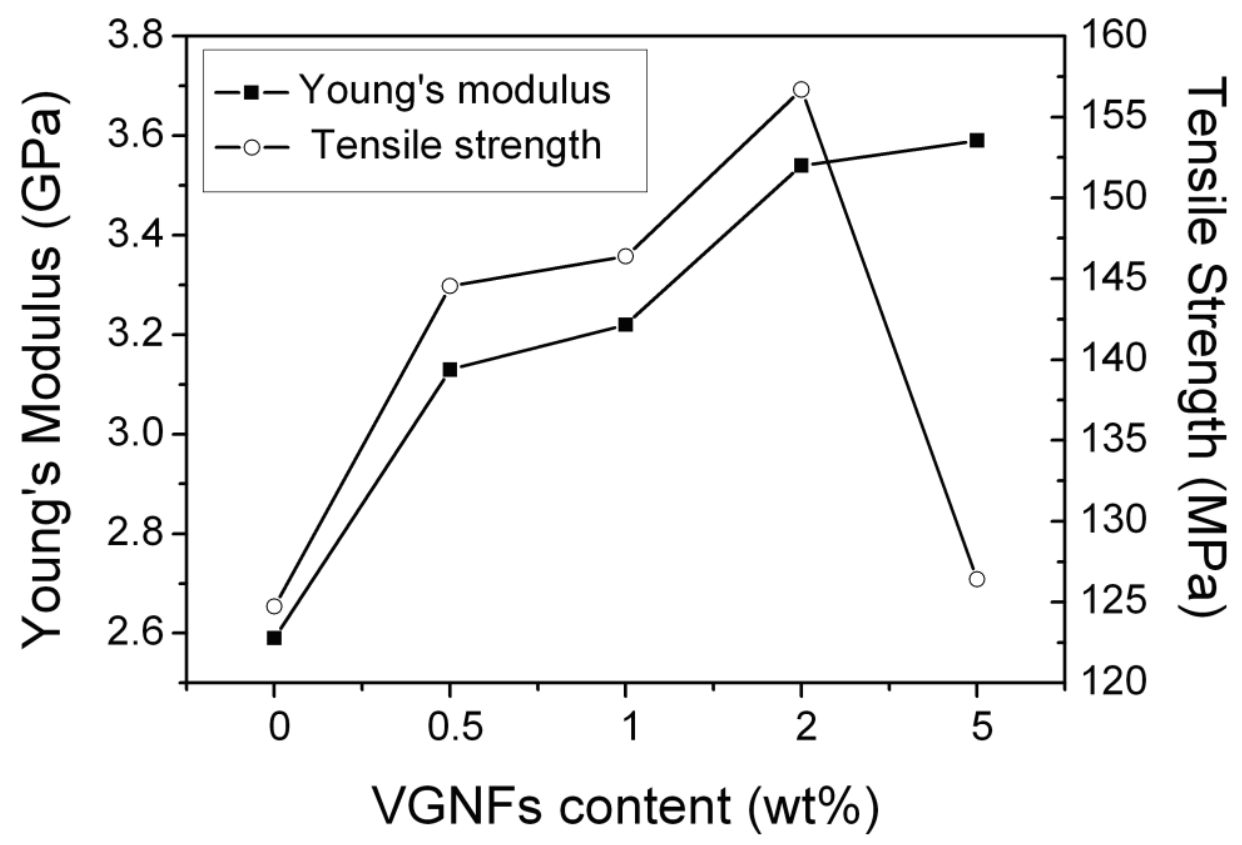

Fig. 4 


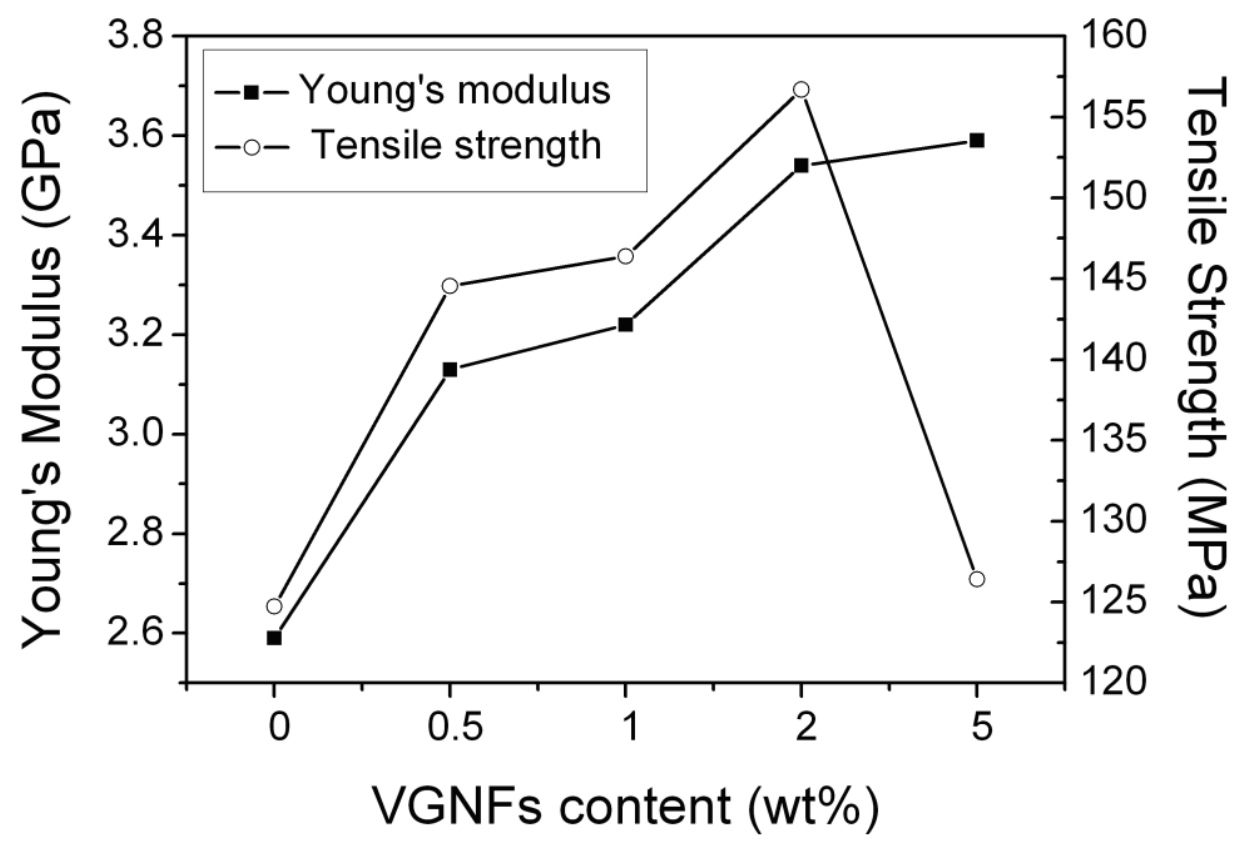

Fig. 5 


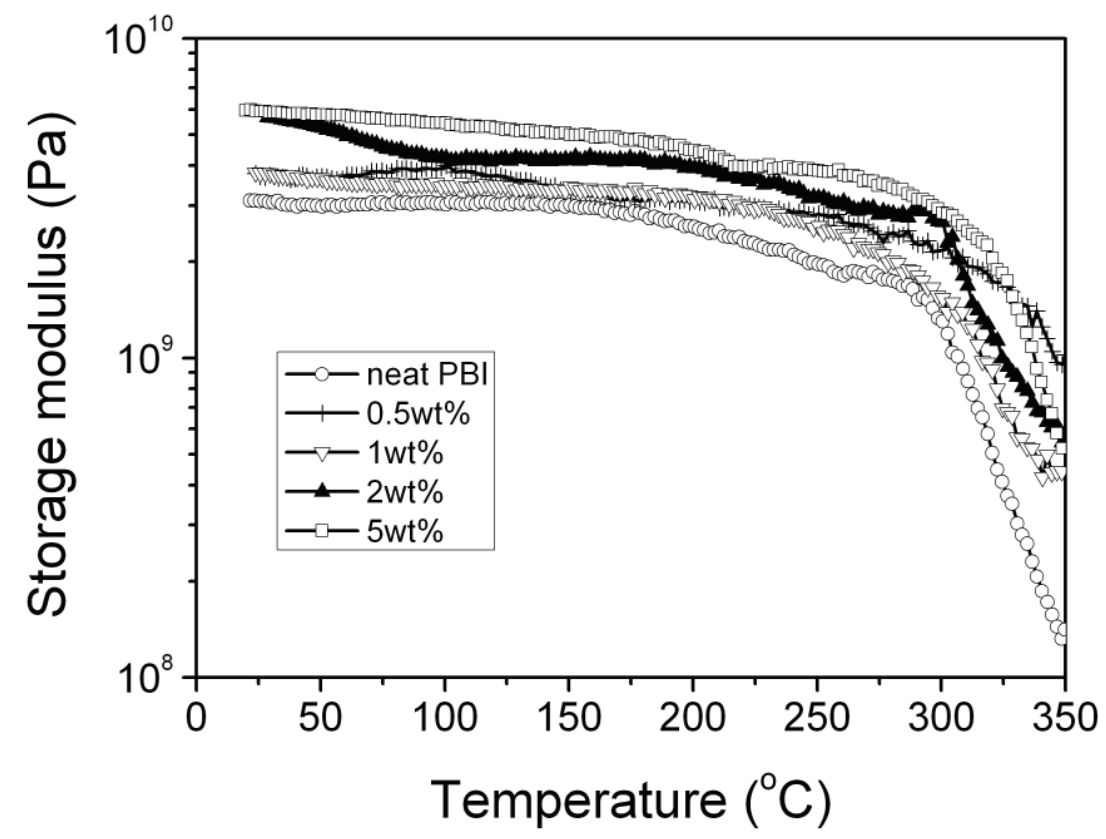

Fig. 6 


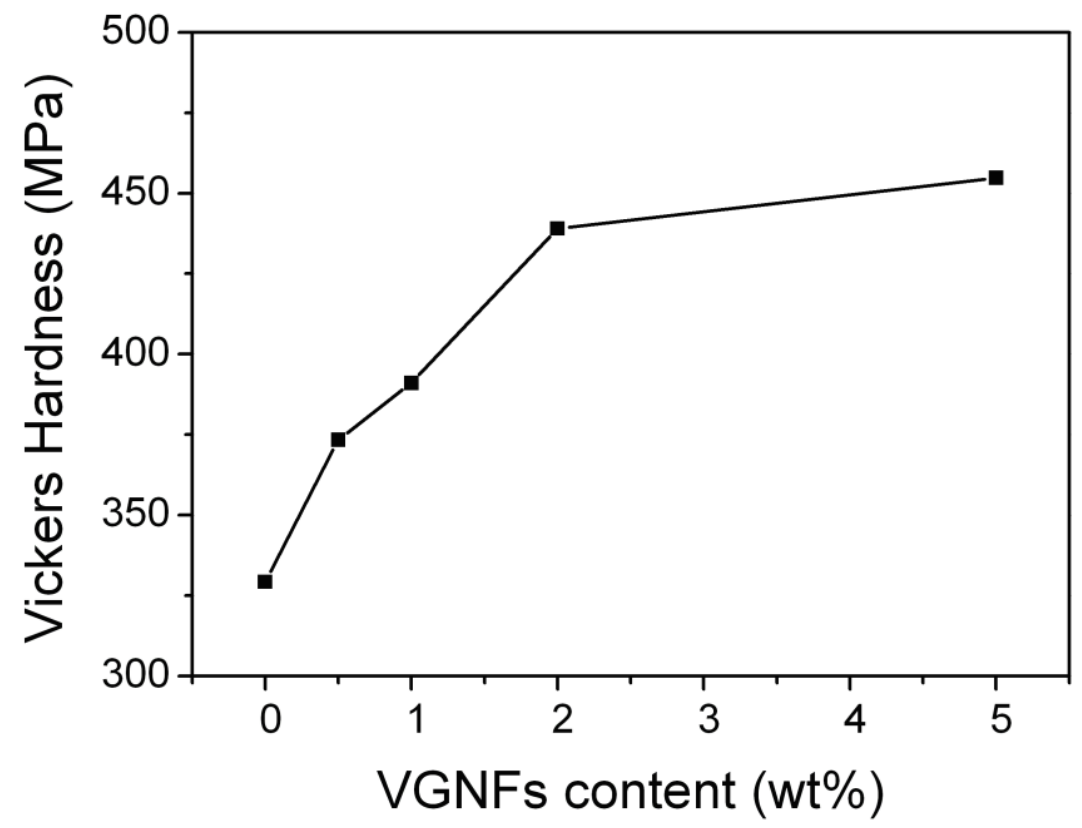

Fig. 7 


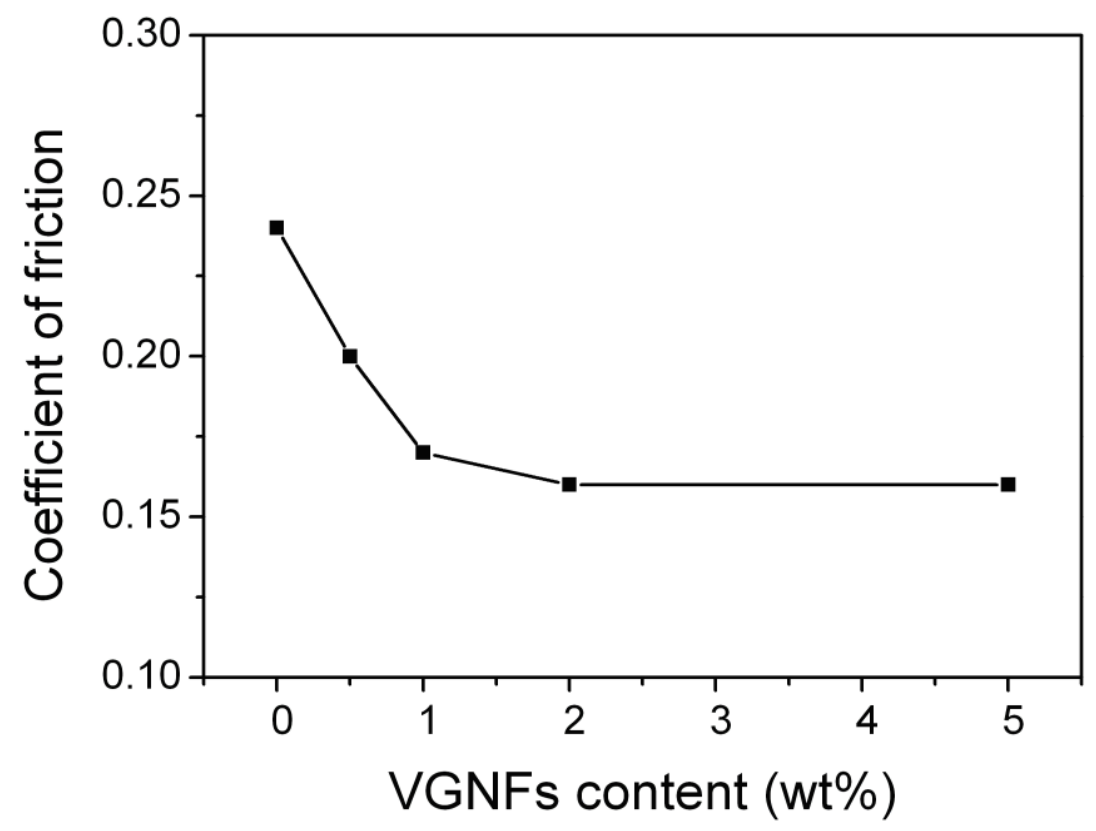

Fig. 8 